\title{
Validity, reliability, and sensitivity to change of the Thai version of the Migraine-Specific Quality of Life Questionnaire version 2.1
}

Thanin Asawavichienjinda*, Pongpat Vorasayan, Jirawadee Noiwattanakul, Kammant Phanthumchinda

Abstract

Background: The Migraine-Specific Quality of Life Questionnaire version 2.1 (MSQv2.1) is used to evaluate the impact of symptoms on the quality of life (QoL) of migraineurs.

Objective: To evaluate primarily the concurrent validity, test-retest reliability, and internal consistency, and secondarily the sensitivity to change of a Thai version of the MSQv2.1.

Methods: The original English version of the MSQv2.1 was translated into a Thai version. The Thai version of the MSQv2.1 was assessed for content and language equivalence. Validity of the Thai version of the MSQv2.1 was assessed using migraine characteristics in a prospective study conducted at the Chulalongkorn Comprehensive Headache Centre of King Chulalongkorn Memorial Hospital. Test-retest reliability and internal consistency were tested in migraineurs. Sensitivity to change was evaluated in another group of migraineurs using an 8-week follow-up.

Results: We recruited 30 migraineurs to test the validity, test-retest reliability, and internal consistency of the Thai version of the MSQv2.1 and 11 migraineurs to test its sensitivity to change. The Thai version of the MSQv2.1 scores were significantly correlated with migraine symptoms (inverse coefficient range from -0.62 to -0.39 ) except for associated symptoms, which had no correlation with any of the dimensions or overall QoL score. Spearman's correlation coefficient for test-retest reliability was $0.56-0.83$, and Cronbach's $\alpha$ for internal consistency was $0.91-$ 0.96. Headache, including average pain duration per attack, pain severity score (numeric rating scale), associated symptoms and dimensions, and overall QoL score of the Thai version of MSQv2.1 improved over time $(P<0.05)$. Moreover, improvement in headache correlated (coefficient range 0.67-0.77) with improvement in overall QoL score and some dimensions of the Thai version of the MSQv2.1 (coefficient range 0.66-0.77).

Conclusion: The Thai version of the MSQv2.1 had validity, acceptable internal consistency, moderate-to-strong test-retest reliability, and strong correlation between improvement in headache severity and overall QoL score. A future study with a larger sample size and longer follow-up is required for better estimates of internal consistency and sensitivity to change.

Keywords: health-related quality of life, Migraine-Specific Quality of Life Questionnaire version 2.1, reliability, sensitivity to change, Thai version of the MSQv2.1, validity

*Correspondence to: Thanin Asawavichienjinda, Department of Medicine, Faculty of Medicine, King Chulalongkorn Memorial Hospital, Chulalongkorn University, Bangkok 10330, Thailand, e-mail:Thanin.A@chula.ac.th

Department of Medicine, Faculty of Medicine, King Chulalongkorn Memorial Hospital, Chulalongkorn University, Bangkok 10330, Thailand

(c) (1) () $\Theta(2017$ Thanin Asawavichienjinda, Pongpat Vorasayan, Jirawadee Noiwattanakul, Kammant Phanthumchinda

This Work is licensed under Creative Common License 
Migraine is a common neurological disorder characterized by unilateral throbbing headache with moderate-to-severe pain intensity lasting for 4-72 $\mathrm{h}$ and aggravated by head motion, bright light, and noise. Migraine is also associated with nausea and vomiting [1]. These migraine symptoms lead to substantial disability, including physical, psychological, and social aspects [2]. Moderate-to-severe head pain of migraine and its associated symptoms during attacks disable and reduce the ability of migraineurs to work and function effectively. The migraine attack interval, even during the headache-free period, still causes migraineurs to be frustrated, worried, and afraid of upcoming migraine attacks. Migraineurs may be reluctant to participate in social activities that may trigger their migraine attacks. Migraine therefore has a significant impact on the quality of life (QoL) of migraineurs [3]. Most migraineurs will seek medicine to terminate pain during the attack and some to prevent attacks if necessary [4].

Currently, there are at least 7 scales to measure QoL, specifically for people with migraine, and these scales can be categorized into 3 groups. The first group is applied to adults and includes (1) the QoL questionnaire created ad hoc by Cavallini et al. without any validity and reliability test [5], (2) the Migraine-Specific Quality of Life Questionnaire (MSQOL) for long-term impact of migraine without a specific period [6], (3) the MSQ developed by Jhingran et al. from GlaxoWellcome for long-term impact of migraine for a specific period of 4 weeks [7], (4) the 6-item Headache Impact Test (HIT-6) for nonspecific type of headache including migraine at the initial development [8], and (5) the Comprehensive Headache-Specific Quality of Life Questionnaire for migraine and tension-type headache only tested for validity [9]. The second group is applied to adolescents only, and uses the (6) Quality of Life Headache in Youth (QLH-Y) scale for chronic headache, including migraine with a 1 week impact [10]. The third group is applied to measure the efficacy of acute treatment and uses the brief 24-hour (7) MSQ for short-term impact [11]. There have been other questionnaires measuring some parts of the QoL domains, such as the Headache Disability Inventory, Headache Impact Questionnaire, and Migraine Disability Assessment (MIDAS) questionnaire.

Migraine-specific QoL questionnaires that are most commonly used are the MSQ and HIT-6. The MSQ was first developed using a 4-phase approach, which included item selection starting with 48 items relevant to migraine, item reduction with 25 items retained, pretesting, and item finalization with 16 items covering 3 dimensions, and a test for validity and reliability [7]. This version of questionnaire is known as version 1.0. After clinical testing in people with episodic migraine, revisions were made to improve discriminant and convergent validity and reliability $[12,13]$. This revised version of the questionnaire is known as version 2.0. The factorial structure of the version 2.0 of the MSQ was then evaluated by using confirmatory factor analysis $[13,14]$, and the results determined a 14 -item version of the statistically improved MSQ [13]. This revised version is known as MSQ version 2.1 (MSQv2.1), and is composed of 14 items in 3 dimensions covering functional status specific to migraine role restrictive (RR), role preventive (RP), and emotional function (EF) [13]. Each item of the MSQv2.1 has a 6-point frequency scale ranging from none of the time, a little bit of the time, some of the time, a good bit of the time, most of the time, and all of the time, which are assigned scores of $1-6$, respectively [15]. Construct validity was also tested with migraine symptoms with low-to-modest correlations $(r=0.1-0.4)$ [13]. The reliability of the questionnaire was tested with Cronbach's $\alpha$ ranging from 0.86 to 0.96 and with intraclass correlation coefficients ranging from 0.57 to 0.63 across the 3 dimensions [13]. For validity and reliability, the MSQv2.1 was widely tested using a diversity of patients, including those with chronic migraine [15] and those with prophylactic migraine treatment [16]. The MSQv2.1 has also been tested for sensitivity to change in clinical trials including those for prophylactic treatment with topiramate in chronic migraine [17, 18]. In clinical trials, improvement in mean number of days per month of headache [17] and at least a $25 \%$ mean reduction in migraine days [18] was concordant with an improvement in RR and EF dimensions of the treatment group.

The HIT-6 was originally composed of 54 items and was analyzed using an item response theory. Subsequently, 10 candidate items were selected and 35 more items were added as suggested by clinicians. This questionnaire was evaluated for clinical validity by telephone interview of people sampled from a migraine medication prescription database who were prescribed medication in the previous year. The people in the migraine medication prescription database might not all be people with migraine. Headache including other types of primary headache can be relieved by migraine medications. Six items then were selected for the new HIT short form (HIT-6) and were again tested for construct validity, including convergent validity and discriminant validity, and for reliability, including internal consistency and test-retest reliability [8]. The validity and reliability of the HIT-6 was also tested on participants with episodic and chronic migraines [19]. In addition, the HIT-6 was tested for chronic migraine [20] and applied in both clinical research $[21,22]$ and clinical practice $[23,24]$. However, one clinical research study demonstrated that topiramate significantly reduced the mean number of monthly migraine days compared with placebo, but no significant intergroup difference was found using HIT-6 [21]. 
The other study demonstrated that botulinum toxin significantly improved headache severity and had improved HIT-6 score compared with placebo [22]. Among these 2 studies, one study did not evaluate how sensitive the score was to change [23]. The other study demonstrated that headache severity correlated with HIT-6 scale score; however, this study included people with episodic migraine, migrainous disorders, and other forms of episodic headache [24].

Not all migraine characteristics have clinically important outcomes. QoL assessment is a clinically important outcome for assessing a treatment effect. If the migraine characteristics improve, the MSQv2.1 score also improves, and this is known as sensitivity to change.

In Thailand, there were no specific questionnaires to evaluate QoL for people with migraine. The present study had 2 objectives. The primary objective aimed to evaluate concurrent validity and test-retest reliability of the Thai version of MSQv2.1. The secondary objective aimed to evaluate internal consistency and sensitivity to change of the Thai version of MSQv2.1 as a pilot study.

\section{Materials and methods}

This was a prospective study conducted at the Chulalongkorn Comprehensive Headache Centre of King Chulalongkorn Memorial Hospital, Faculty of Medicine, Chulalongkorn University, in 2009 and was approved by the institutional review board of Faculty of Medicine, Chulalongkorn University (No. 034/52).

\section{Patients}

Two groups of patients were included in the present study. One group was used to test for concurrent validity, test-retest reliability, and internal consistency of the Thai version of MSQ 2.1, and the other group was used to test clinical sensitivity of the Thai version of MSQ 2.1 to change. Both groups of patients were those who visited the Chulalongkorn Comprehensive Headache Centre and were diagnosed as having migraine with or without aura. The inclusion criteria were patients aged 18-65 years who were literate in Thai and gave their written informed consent to participate in this study after receiving an invitation in a consecutive manner. Migraine diagnostic criteria and classification were based on the International Classification of Headache Disorders, first or second edition, that depended on headache characteristics received before or after $2004[25,26]$.

\section{Concurrent validity test of the Thai version of MSQv2.1}

We received permission from GSK to use the original English questionnaire of the MSQv2.1 for translation and for use and validation of the Thai version. The MSQv2.1 was translated into Thai by the first author (TA). The following process was conducted to validate the Thai version of MSQv2.1 as equivalent to the original English version of MSQv2.1. The Thai version was translated back into English by a person with a high degree of English proficiency who was blinded to the original version. Subsequently, the original English version and the English version from the back translation were compared. If at any point the 2 English versions were different, the processes of translation into Thai and back translation into English were repeated until both English versions were identical in content, so-called "content equivalence". Ultimately, the Thai version of MSQv2.1 was used with cultural modifications by an active informant experienced with migraine headache to simplify the Thai wording for ease of understanding and application. This process was called language equivalence. The Thai version of MSQv2.1 was then tested for concurrent validity with migraine characteristics including frequency of attacks per 4 weeks, average pain during per attack (measured in hours), average pain severity score measured using a numerical rating scale (NRS), and associated symptoms and headache severity in the previous 4 weeks. The headache severity was on a scale of mild (does not inhibit work or other activities), moderate (inhibits but does not prohibit), or severe (prohibits work and other activities). Each item on the Thai version of MSQv2.1 had a 6-point scale ranging from none of the time, a little bit of the time, some of the time, a good bit of the time, most of the time, and all of the time, which were assigned scores of $1-6$, respectively, corresponding to the original version [15]. Raw dimension scores for RR, RP, and EF were computed as the sum of item responses, and overall QoL score were computed as the sum of the total item responses, and all were rescaled to a $0-100$ scale, where higher scores indicated a better health-related QoL [15].

\section{Reliability test for test-retest reliability and internal consistency of the Thai version of MSQv2.1}

The Chulalongkorn Comprehensive Headache Centre of King Chulalongkorn Memorial Hospital has been established since 2000. This center registers every patient who visits and records every visit using a standardized patient record form. There are 2 types of patient record forms: initial and follow-up. 
The initial form records patient demographics and socioeconomic data and history of headache including frequency (episodes per 4 weeks), pain duration (hours), pain severity score measured using NRS [zero (no pain) to 10 (maximum pain)], associated symptoms and headache severity, comorbidity, and previous treatments. The follow-up form records the history of headache, efficacy, adverse effects of acute pain/headache prophylactic medications (if any), and current treatments. The center also treats patients individually with prescription for acute pain and prophylactic medications (if any) and gives them specific advice for their migraine management problems. The patients also receive a headache diary that is used to educate them. The diary contains information on headache characteristics that can differentiate primary from secondary headache, the characteristics of migraine headache, a list of precipitating factors, how to control pain during an attack with nonpharmacological and pharmacological treatments, how to prevent pain with nonpharmacological and pharmacological treatments, an explanation of medication overuse and why compliance with medications is needed, and a schedule for follow-up.

The Thai version of MSQv2.1 was applied to migraine patients in a consecutive manner. The participants completed the first questionnaire by self-administration. The first questionnaire included 2 parts. The first part asked about their history of headache in the previous 4 weeks, and the second part was the Thai version of MSQv2.1. Before patients left the center, they received a second questionnaire to be completed at home within the following 2 weeks. The second questionnaire was similar to the first questionnaire with the exception of an additional question inquiring about whether the QoL at the time of the first and second questionnaires was the same. Enclosed along with the blank second questionnaires were stamped envelopes addressed to the third author $(\mathrm{JN})$ for participants to conveniently mail back the second questionnaire after completion. At 2 weeks from the time of the first completed questionnaire, the third author (JN) reminded participants via telephone call to complete and return the second questionnaire. Standard post in Thailand takes an average of 2 weeks for delivery. Therefore, if the second questionnaire was not received or in the event that the received second questionnaire was incomplete or the answer was different in the additional question, the other consecutively eligible patients at the center were invited to replace previous patients until the number of participants met the assigned sample size. For the reliability test, the test-retest reliability was compared between the first and the second completed Thai version of MSQv2.1. The internal consistency of the 2 completed Thai versions of MSQv2.1 was tested for each occasion.

\section{Clinical testing to evaluate sensitivity of the Thai version of MSQv2.1 to change}

After tests for concurrent validity, test-retest reliability, and internal consistency of the Thai version of MSQv2.1 were complete, the Thai version of MSQv2.1 was then tested for sensitivity to change to history of headache in another group of migraineurs at the Chulalongkorn Comprehensive Headache Centre. The history of headache of the people who visited the center was applied at an arbitrary point that we called time zero and was followed for 4 weeks (so-called "week 4 application") and 8 weeks (so-called "week 8 application"). The history of headache and the Thai version of MSQv2.1 scores including those for RR, RP, and EP and overall QoL score were compared between 2 periods: week 4 and time zero, week 8 and time zero, and week 8 and week 4 . The difference in the history of headache between the 2 periods was compared to difference in scores for the dimensions RR, RP, and EF and overall QoL score of the Thai version of MSQv2.1 between the 2 periods to test whether or not the Thai version of MSQv2.1 was sensitive to change.

\section{Statistical analysis}

Concurrent validity of the Thai version of MSQv2.1 was tested with migraine characteristics using Spearman's correlation coefficient for ordinal data and a rank-biserial correlation coefficient for correlation between nominal and ordinal data. Reliability of the questionnaire was tested using Spearman's correlation coefficient for test-retest reliability and using Cronbach's $\alpha$ for internal consistency. The history of headache 4 weeks before completion of the first and second questionnaires and the scores of the Thai version of MSQv2.1 were summarized with median and interquartile range. Associated symptoms and adverse effects of acute pain or prophylactic medications were categorized into dichotomous data on the basis of "yes" or "no" answers. The headache severity with the scale of mild (does not inhibit work or other activities), moderate (inhibits but does not prohibit), or severe (prohibits work and other activities) was categorized into dichotomous data as follows: mild and moderate or severe, with data summary of percentage. Clinical testing for the difference in the history of headache and the QoL 
scores between 2 periods was conducted using the McNemar $\chi^{2}$ or sign test according to the type of the data. Sensitivity to change in each of the 2 periods between the difference in the history of headache and the difference in the dimension scores or overall QoL score of the Thai version of MSQv2.1 was tested using a rank-biserial correlation coefficient for analysis of nominal and ordinal data and a Spearman's correlation coefficient for analysis of ordinal and ordinal data. $P<0.05$ was considered as significant. However, a corrected $P$ for multiple analyses was not used. SPSS Statistics for Windows (version 21; IBM Corporation) was used for all statistical analyses.

The sample size for test-retest reliability was based on the Spearman's correlation coefficient with a type I error of 0.05 and power of 0.88 (equivalent to 0.8 for a Pearson correlation coefficient), and anticipated correlation coefficient of 0.5 was calculated according to Siegel's book of nonparametric statistics [27]. Therefore, the total sample size required was 29 participants. We did not calculate the sample size for the internal consistency and clinical testing for sensitivity to change. These were analyzed in a pilot study.

\section{Results}

There were 46 participants who completed the first questionnaire. A total of 37 participants mailed back the second questionnaire. Of them, 30 participants reported the same QoL between the first and second questionnaires and were eligible for reliability test analyses (Figure 1); 29 (97\%) participants were females with a mean age of 42.4 years [standard deviation (SD) 13.4 years] (Table 1). Of them, 11 (37\%) participants had upper
Table 1. Baseline characteristics of the 30 eligible participants

\begin{tabular}{lr}
\hline Characteristic & Value \\
\hline Age, years (SD) & $42.4(13)$ \\
Female sex, $\mathrm{n}(\%)$ & $29(97)$ \\
Educational level $^{\dagger}, \mathrm{n}(\%)$ & \\
$\quad$ Primary education & $8(27)$ \\
$\quad$ Upper secondary education & $3(10)$ \\
Postsecondary nontertiary education & $5(17)$ \\
First and second stage of tertiary education & $14(47)$ \\
Classification of migraine, $\mathrm{n}(\%)$ & \\
$\quad$ Migraine without aura & $27(90)$ \\
$\quad$ Migraine with aura & $3(10)$ \\
Duration of having migraine (years) & \\
$\quad$ Median & 5.3 \\
Interquartile range & 8.0 \\
Average pain duration per attack (hours) & 3.8 \\
$\quad$ Median & 4.0 \\
Interquartile range & $1(3)$ \\
Average pain severity, $\mathrm{n}(\%)$ & $23(77)$ \\
Mild & $6(20)$ \\
Moderate & \\
Severe & $19(63)$ \\
Associated symptoms ${ }^{\ddagger}, \mathrm{n}(\%)$ & $17(57)$ \\
Nausea & $15(50)$ \\
Photophobia & $13(43)$ \\
Vomiting & \\
\hline
\end{tabular}

'Based on UNESCO's International Standard Classification of Education $1997,{ }^{\ddagger}$ Counted more than once

SD, standard deviation; UNESCO, United Nations Educational, Scientific and Cultural Organization

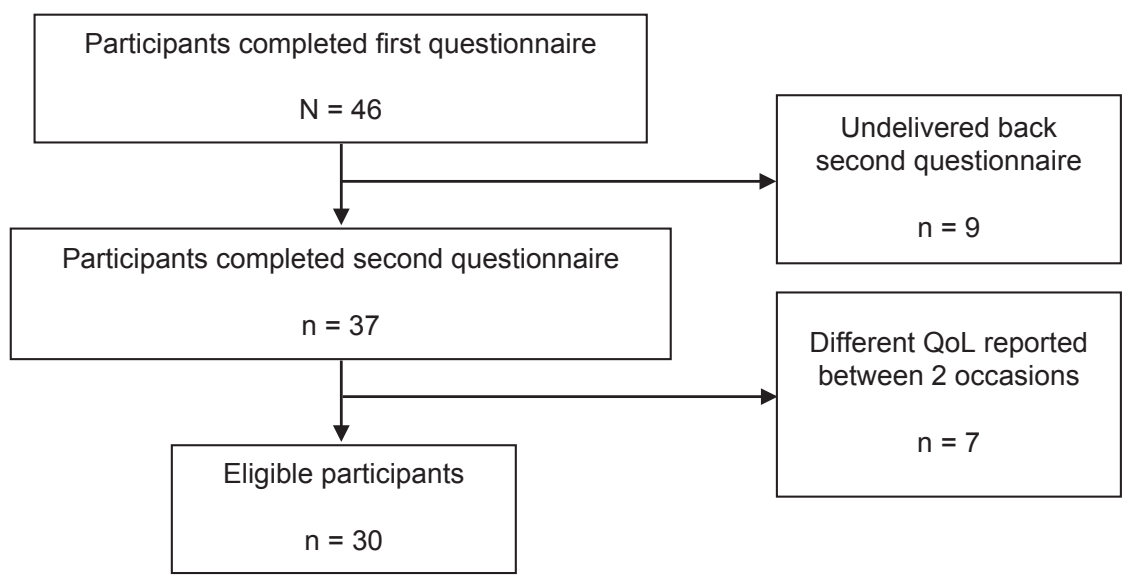

Figure 1. Flow diagram for recruitment of participants to test for concurrent validity, test-retest reliability, and internal consistency of the Thai version of the Migraine-Specific Quality of life Questionnaire version 2.1; QoL, quality of life 
Table 2. History of headache 4 weeks before completion of the first and second questionnaires

\begin{tabular}{|c|c|c|c|}
\hline $\begin{array}{l}\text { Headache information/score } \\
\text { of Thai version of the MSQv2.1 }\end{array}$ & $\begin{array}{r}\text { First } \\
\text { questionnaire }\end{array}$ & $\begin{array}{r}\text { Second } \\
\text { questionnaire }\end{array}$ & $P$ \\
\hline \multicolumn{4}{|l|}{$\begin{array}{l}\text { Frequency of attacks } 4 \text { weeks } \\
\text { before (episodes) }\end{array}$} \\
\hline Median & 4.0 & 4.0 & NS \\
\hline Interquartile range & 7.2 & 7.0 & \\
\hline \multicolumn{4}{|l|}{$\begin{array}{l}\text { Average pain duration } \\
\text { per attack (hours) }\end{array}$} \\
\hline Median & 2.0 & 2.0 & NS \\
\hline Interquartile range & 3.6 & 2.9 & \\
\hline \multicolumn{4}{|l|}{$\begin{array}{l}\text { Average pain severity score } \\
\text { (NRS) }\end{array}$} \\
\hline Median & 4.5 & 5.0 & NS \\
\hline Interquartile range & 4.0 & 5.2 & \\
\hline $\begin{array}{l}\text { No. of associated } \\
\text { symptoms, } n(\%)\end{array}$ & $15.0(50)$ & $16.0(53)$ & NS \\
\hline \multicolumn{4}{|l|}{ Headache severity, n (\%) } \\
\hline Mild & $22(73)$ & $20(67)$ & NS \\
\hline Moderate or severe & $8(27)$ & $10(33)$ & \\
\hline
\end{tabular}

MSQv2.1, Migraine-Specific Quality of Life Questionnaire version 2.1; NRS, numerical rating scale; NS, not significant

secondary education or less. Most participants (90\%) were classified as having migraine without aura. The median duration of having migraine was 5.3 years (interquartile range 8.0), and pain duration per attack was 3.8 hours (interquartile range 4.0) with or without taking acute pain medications. Approximately $75 \%$ of participants had moderate pain intensity and $20 \%$ had severe pain intensity. In all, $\geq 50 \%$ of the pain was accompanied by nausea (63\%), photophobia (57\%), or phonophobia (50\%). History of headache 4 weeks before completing the first and second questionnaires is displayed in Table 2. Findings were almost identical including frequency of attacks, average pain duration, average pain severity score, and associated symptoms except headache severity, with a slightly higher score for mild degree in the first questionnaire (73\% vs $67 \%$ ). The concurrent validity of the Thai version of MSQv2.1 as shown in Table 3 had a significant reverse correlation with the migraine characteristics; correlation coefficient ranged from -0.62 to -0.39 except average pain duration and RP, headache severity and RR, and associated symptoms not associated with any of the dimensions of the Thai version of MSQv2.1. This meant that the more frequent the headache, pain duration, pain severity, and headache severity of the participants, the less QoL they perceived. The correlation of scores, 1-6, for each item in the Thai version of MSQv2.1, between the first and the second questionnaires, was tested with a Spearman's correlation coefficient of $>0.60$, with the exception of items
Table 3. Concurrent validity of the Thai version of MSQv2.1 score with the migraine characteristics 4 weeks before completion of the first questionnaire

\begin{tabular}{|c|c|c|c|c|}
\hline \multirow[t]{2}{*}{ Migraine characteristic } & \multicolumn{4}{|c|}{ Thai version of the MSQv2.1 score } \\
\hline & RR & RP & EF & Overall QoL \\
\hline Frequency of attacks & $-0.42^{*}$ & $-0.41^{*}$ & $-0.39 *$ & $-0.50^{*}$ \\
\hline 4 weeks before (episodes) & & & & \\
\hline $\begin{array}{l}\text { Average pain duration per } \\
\text { attack (hours) }\end{array}$ & $-0.47^{*}$ & -0.34 & $-0.48^{*}$ & $-0.48^{*}$ \\
\hline $\begin{array}{l}\text { Average pain severity score } \\
\text { (NRS) }\end{array}$ & $-0.58^{*}$ & $-0.53^{*}$ & $-0.50^{*}$ & $-0.62^{*}$ \\
\hline No. of associated symptoms & 0.24 & 0.35 & 0.29 & 0.30 \\
\hline $\begin{array}{l}\text { Mild degree of headache } \\
\text { severity }\end{array}$ & -0.27 & $-0.43^{*}$ & $-0.50^{*}$ & $-0.42^{*}$ \\
\hline
\end{tabular}

*P $P<0.05$

EF, emotional function; MSQv2.1, Migraine-Specific Quality of Life Questionnaire version 2.1; NRS, numerical rating scale; QoL, quality of life; $R P$, role function - preventive; $R R$, role function - restrictive

2 and 14 with correlation coefficients of 0.58 and 0.56 , respectively (Table 4). The correlations of scores of the RR, RP, and EF dimensions and the overall QoL score between the first and second questionnaires had a coefficient range from 0.65 to 0.83. Cronbach's $\alpha$ for internal consistency for each of the first and the second questionnaires ranged from 0.91 to 0.96 .

Clinical testing of 12 patients at the center with a diagnosis of migraine was conducted to determine the sensitivity of the Thai version of MSQv2.1 to change. Of the 12 patients, 1 patient was lost to follow-up. Data from 11 migraineurs were ultimately included for analysis (a flow diagram is shown in Figure 2). Baseline characteristics of the included 11 patients are summarized in Table 5. There were 10 female participants with mean age of 39.6 years (SD 14.7). Five participants had equivalent to upper secondary education or less, and most of them $(73 \%)$ were employed. The median duration of having a migraine was 3 years with an interquartile range of 8.0 years. After 8 weeks of treatment and follow-up (Table 6), the frequency of attacks per 4 weeks dramatically reduced from a median of 12 attacks at time zero to a median of 8 attacks at week 4 and 2 attacks at week 8 , but we did not find a significant difference. Average pain duration per attack reduced from a median of 3 to 1.5 hours and then to 1 hour with a significant difference between week 8 and time zero $(P=0.002)$ and between weeks 8 and $4(P=0.02)$. Average pain severity score also significantly reduced from a median of 6 at time zero to 5 in week $4(P=0.04)$. The pain appeared to stabilize in week 8 with a median pain severity score of 5 . No significant difference in average pain severity score was found between week 8 and time zero or between weeks 8 and 4 . 
Table 4. Score of each item and domain of the Thai version of the MSQv2.1 between the first and second questionnaires

\begin{tabular}{|c|c|c|c|c|}
\hline Score of Thai version of the MSQ 2.1 & First questionnaire & Second questionnaire & $r^{+}$ & $P$ \\
\hline \multicolumn{5}{|l|}{ RR - median score (interquartile range) } \\
\hline $\begin{array}{l}\text { Item } 1 \text { family interfered with how well you dealt with family, } \\
\text { friends, and others }\end{array}$ & $3.5(2.2)$ & $4.0(2.0)$ & 0.68 & $<0.001$ \\
\hline $\begin{array}{l}\text { Item } 2 \text { leisure interfered with your leisure time activities, such as } \\
\text { reading and exercising }\end{array}$ & $3.0(3.0)$ & $4.0(2.0)$ & 0.58 & 0.001 \\
\hline Item 3 activity - had difficulty in performing work or daily activities & $4.0(2.2)$ & $4.0(2.0)$ & 0.77 & $<0.001$ \\
\hline Item 4 work - kept you from getting as much done at work or at home & $4.0(2.0)$ & $3.5(2.0)$ & 0.66 & $<0.001$ \\
\hline Item 5 concentration - limited your ability to concentrate on & $3.5(3.0)$ & $4.0(2.2)$ & 0.61 & $<0.001$ \\
\hline \multicolumn{5}{|l|}{ work or daily activities } \\
\hline Item 6 tired - left you too tired to do work or daily activities & $3.0(2.3)$ & $4.0(2.0)$ & 0.71 & $<0.001$ \\
\hline Item 7 energy - limited the number of days you have felt energetic & $3.0(2.2)$ & $4.0(2.0)$ & 0.72 & $<0.001$ \\
\hline RR dimension scores (rescale to $0-100$ ) - median (interquartile range) & $71.4(39.2)$ & $77.1(23.6)$ & 0.83 & $<0.001$ \\
\hline \multicolumn{5}{|l|}{$\mathrm{RP}$ - median score (interquartile range) } \\
\hline Item 8 cancel - cancel work or daily activities & $4.0(2.0)$ & $4.0(3.0)$ & 0.65 & $<0.001$ \\
\hline Item 9 help - need help in handling routine tasks & $5.0(1.0)$ & $4.0(1.0)$ & 0.60 & $<0.001$ \\
\hline Item 10 stop - stop work or daily activities & $4.0(2.0)$ & $4.0(2.0)$ & 0.62 & $<0.001$ \\
\hline Item 11 social - not able to go to social activities & $4.0(2.0)$ & $4.5(2.0)$ & 0.66 & $<0.001$ \\
\hline RP dimension scores (rescale to $0-100$ ) - median (interquartile range) & $87.5(35.0)$ & $80.0(35.0)$ & 0.76 & $<0.001$ \\
\hline \multicolumn{5}{|l|}{ EF - median score (interquartile range) } \\
\hline Item 12 frustrated - felt fed up or frustrated & $3.5(3.0)$ & $4.0(2.0)$ & 0.68 & $<0.001$ \\
\hline Item 13 burden - felt like you were a burden on others & $5.0(2.0)$ & $4.0(1.0)$ & 0.68 & $<0.001$ \\
\hline Item 14 afraid - afraid of letting others down & $5.0(1.0)$ & $5.0(1.0)$ & 0.56 & 0.001 \\
\hline EF dimension scores (rescale to $0-100$ ) - median (interquartile range) & $80.0(30.0)$ & $83.3(21.6)$ & 0.65 & $<0.001$ \\
\hline Overall QoL score (rescale to 0-100) - median (interquartile range) & $76.7(29.3)$ & $80.0(24.6)$ & 0.83 & $<0.001$ \\
\hline
\end{tabular}

${ }^{\dagger}$ Spearman's correlation coefficient

EF, emotional function; MSQv2.1, Migraine-Specific Quality of Life Questionnaire version 2.1; QoL, quality of life; RP, role function - preventive; RR, role function - restrictive

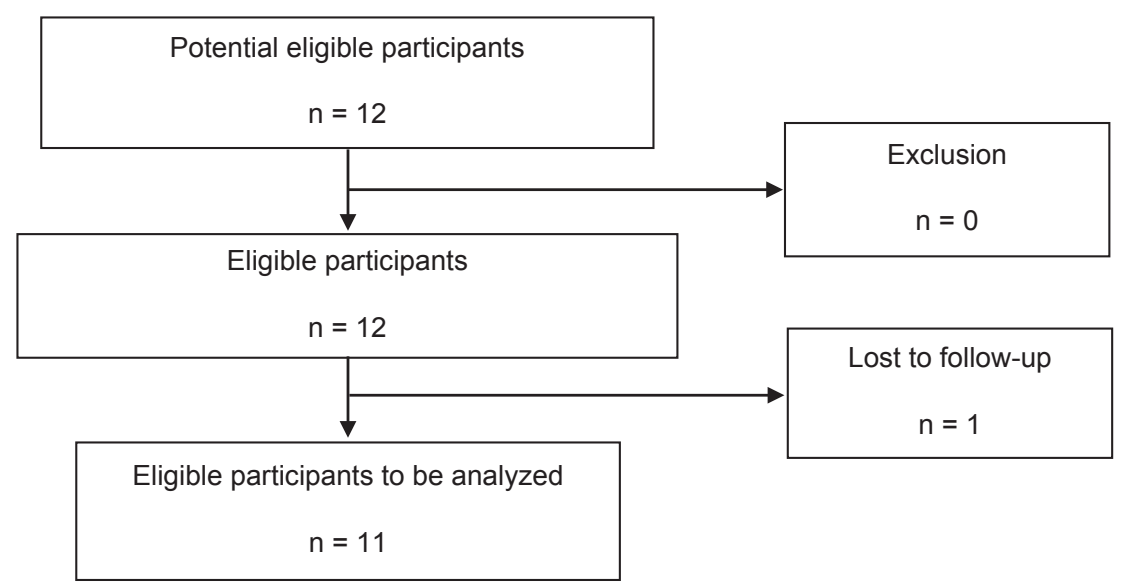

Figure 2. Flow diagram for recruitment of participants to test for sensitivity of the Thai version of the Migraine-Specific Quality of Life Questionnaire version 2.1 to change

Headache severity was reduced to mild with a higher proportion in week $4(82 \%)$ and week $8(100 \%)$ compared with time zero (55\%), although the reductions were not significant. Migraineassociated symptoms, nausea or vomiting, and photophobia or phonophobia significantly decreased from time zero $(82 \%$ and $91 \%$, respectively) to week 4 (18\% and $18 \%$, respectively) and week 8 (9\% and $27 \%$, respectively) with a significant difference between week 4 and time zero $(P=0.01$ and $P=0.002$, respectively) and between week 8 and time zero ( $P=0.002$ and $P=0.007$, respectively). The proportion of 
Table 5. Baseline characteristics of the 11 participants for clinical testing

\begin{tabular}{lr}
\hline Characteristic & Value \\
\hline Age, years (SD) & $39.6(14.7)$ \\
Female sex, $\mathrm{n}(\%)$ & $10(91)$ \\
Educational level, $\mathrm{n}(\%)$ & \\
$\quad$ Primary education & $2(18)$ \\
Upper secondary education & $3(27)$ \\
Postsecondary nontertiary education & $5(46)$ \\
First stage of tertiary education & $1(9)$ \\
Occupation, $\mathrm{n}(\%)$ & \\
Employee & $7(64)$ \\
Studying & $3(27)$ \\
Business & $1(9)$ \\
Duration of having migraine, years & \\
$\quad$ Median & 3.0 \\
Interquartile range & 8.0 \\
\hline
\end{tabular}

$\mathrm{SD}$, standard deviation participants taking prophylactic medication increased significantly from time zero (27\%) to week $4(91 \%)$ and week 8 $(100 \%)$ with a significant difference between week 4 and time zero $(P=0.007)$. The type of prophylactic medications prescribed to the majority of patients at the Chulalongkorn Comprehensive Headache Centre was antiepileptic drugs, accounting for $64 \%$ at weeks 4 and 8 . Other history of headache was not significantly different between the 2 periods, including the location of pain, side of head pain, pain quality, precipitating factors, proportion of patients taking acute pain medication, type and effect of acute pain medication, and adverse effect of acute pain or prophylactic medications (Table 6). For the QoL aspects (Table 6), median scores of each domain, RR, RP, and EF, and the overall score of MSQv2.1 improved over time with a significant difference when compared between week 4 and time zero $(P=0.02)$ and between week 8 and time zero $(P=0.002$ and 0.001$)$ except for the EF domain. Between

Table 6. Comparison of the change in the history of headache between 2 periods of 4 weeks and the score change in the Thai version of the MSQv2.1

\begin{tabular}{|c|c|c|c|c|c|c|}
\hline Headache history & Time zero $(0)$ & Week 4 (4) & Week 8 (8) & $\begin{array}{r}P \text { (week } 4 \text { and } \\
\text { time zero) }\end{array}$ & $\begin{array}{r}P \text { (week } 8 \text { and } \\
\text { time zero) }\end{array}$ & $\begin{array}{r}P \text { (weeks } 8 \\
\text { and } 4)\end{array}$ \\
\hline \multicolumn{7}{|c|}{ Frequency of attacks 4 weeks before (episodes) } \\
\hline Median & 12.0 & 8.0 & 2.0 & 0.75 & 0.18 & 0.06 \\
\hline Interquartile range & 12.0 & 18.0 & 11.0 & & & \\
\hline \multicolumn{7}{|c|}{ Average pain duration per attack, hours } \\
\hline Median & 3.0 & 1.5 & 1.0 & 1.00 & $0.002 * *$ & $0.02 *$ \\
\hline Interquartile range & 3.5 & 10.5 & 2.0 & & & \\
\hline \multicolumn{7}{|l|}{ Average pain severity score (NRS) } \\
\hline Median & 6.0 & 5.0 & 5.0 & $0.04 *$ & 0.34 & 1.00 \\
\hline Interquartile range & 1.5 & 4.0 & 6.0 & & & \\
\hline \multicolumn{7}{|l|}{ Headache severity, $\mathrm{n}(\%)$} \\
\hline Mild & $6(55)$ & $9(82)$ & $11(100)$ & 0.38 & 0.06 & 0.50 \\
\hline Moderate or severe & $5(45)$ & $2(18)$ & $0(0)$ & & & \\
\hline \multicolumn{7}{|l|}{ Location of pain ${ }^{\dagger}, \mathrm{n}(\%)$} \\
\hline Ocular & $8(73)$ & $7(64)$ & $3(27)$ & 1.00 & 0.09 & 0.19 \\
\hline Back of neck & $6(55)$ & $3(27)$ & $1(9)$ & 0.38 & 0.06 & 0.58 \\
\hline Forehead & $2(18)$ & $0(0)$ & $2(18)$ & NA & 1.00 & NA \\
\hline Temple & $10(91)$ & $8(73)$ & $8(73)$ & 0.58 & 0.58 & 1.00 \\
\hline Occiput & $8(73)$ & $5(45)$ & $5(45)$ & 0.38 & 0.36 & 1.00 \\
\hline Vertex & $2(18)$ & $0(0)$ & $0(0)$ & NA & NA & NA \\
\hline Whole head & $5(45)$ & $2(18)$ & $1(9)$ & 0.36 & 0.14 & 1.00 \\
\hline \multicolumn{7}{|l|}{ Side $^{\dagger}, \mathrm{n}(\%)$} \\
\hline Unilateral, constant & $2(18)$ & $6(55)$ & $4(36)$ & 0.10 & 0.63 & 0.66 \\
\hline Bilateral & $5(45)$ & $5(45)$ & $2(18)$ & 1.00 & 0.36 & 0.36 \\
\hline Unilateral with alternating & $6(55)$ & $2(18)$ & $3(27)$ & 0.10 & 0.36 & 1.00 \\
\hline Unilateral and then bilateral & $2(18)$ & $0(0)$ & $0(0)$ & NA & NA & NA \\
\hline \multicolumn{7}{|l|}{ Pain quality ${ }^{\dagger}, \mathrm{n}(\%)$} \\
\hline Dull aching/pressing & $4(36)$ & $4(36)$ & $2(18)$ & 1.00 & 0.63 & 0.63 \\
\hline Throbbing & $9(82)$ & $6(55)$ & $6(55)$ & 0.36 & 0.36 & 1.00 \\
\hline Sharp or stabbing & $1(9)$ & $0(0)$ & $3(27)$ & NA & 0.58 & NA \\
\hline \multicolumn{7}{|l|}{ Associated symptoms ${ }^{\dagger}, \mathrm{n}(\%)$} \\
\hline Nausea/vomiting & $9(82)$ & $2(18)$ & $1(9)$ & 0.01 & 0.002 & 1.00 \\
\hline Photophobia/phonophobia & $10(91)$ & $2(18)$ & $3(27)$ & 0.002 & 0.007 & 1.00 \\
\hline
\end{tabular}


Table 6. Comparison of the change in the history of headache between 2 periods of 4 weeks and the score change in the Thai version of the MSQv2.1 (Continued)

\begin{tabular}{|c|c|c|c|c|c|c|}
\hline Headache history & Time zero (0) & Week 4 (4) & Week 8 (8) & $\begin{array}{r}P \text { (week } 4 \text { and } \\
\text { time zero) }\end{array}$ & $\begin{array}{r}P \text { (week } 8 \text { and } \\
\text { time zero) }\end{array}$ & $\begin{array}{r}P(\text { weeks } 8 \\
\text { and } 4)\end{array}$ \\
\hline \multicolumn{7}{|l|}{ Precipitating factors, n (\%) } \\
\hline No & $2(18)$ & $2(45)$ & $5(45)$ & 1.00 & 0.36 & 0.36 \\
\hline \multicolumn{7}{|l|}{ Taking acute pain medication, $\mathrm{n}(\%)$} \\
\hline Yes & $11(100)$ & $11(100)$ & $10(91)$ & NA & NA & NA \\
\hline \multicolumn{7}{|l|}{ Type of acute pain medication, $\mathrm{n}(\%)$} \\
\hline Simple analgesic/NSAIDs & $10(91)$ & $10(91)$ & $10(91)$ & 1.00 & 1.00 & 1.00 \\
\hline Migraine-specific medication & $1(9)$ & $1(9)$ & $1(9)$ & 1.00 & NA & NA \\
\hline \multicolumn{7}{|l|}{ Effect of acute pain medication, $\mathrm{n}(\%)$} \\
\hline Pain improvement & $8(73)$ & $9(82)$ & $9(82)$ & 1.00 & 1.00 & 1.00 \\
\hline \multicolumn{7}{|l|}{ Adverse effect of acute pain medication, $\mathrm{n}(\%)$} \\
\hline Yes & $2(18)$ & $0(0)$ & $0(0)$ & NA & NA & NA \\
\hline \multicolumn{7}{|l|}{ Taking prophylactic medication, n (\%) } \\
\hline Yes & $3(27)$ & $10(91)$ & $11(100)$ & 0.007 & NA & NA \\
\hline \multicolumn{7}{|l|}{ Type of prophylactic medication, $\mathrm{n}(\%)$} \\
\hline Antiepileptic drugs & $1(9)$ & $7(64)$ & $7(64)$ & 0.02 & 0.02 & 1.00 \\
\hline Calcium antagonists & $2(18)$ & $1(9)$ & $1(9)$ & 1.00 & 1.00 & 1.00 \\
\hline Antidepressants & $0(0)$ & $2(18)$ & $3(27)$ & NA & NA & 1.00 \\
\hline \multicolumn{7}{|l|}{ Adverse effect of prophylactic medication, $\mathrm{n}(\%)$} \\
\hline Yes & $0(0)$ & $2(18)$ & $1(9)$ & NA & NA & 1.00 \\
\hline \multicolumn{7}{|l|}{$\begin{array}{l}\text { Adverse effect of both acute pain and } \\
\text { prophylactic medications, } \mathrm{n}(\%)\end{array}$} \\
\hline Yes & $2(18)$ & $2(18)$ & $1(9)$ & 1.00 & 1.00 & 1.00 \\
\hline $\mathrm{RR}$ - median score (interquartile range) & $48.6(28.6)$ & $85.7(22.9)$ & $85.7(20.0)$ & 0.02 & 0.002 & 0.06 \\
\hline $\mathrm{RP}$ - median score (interquartile range) & $70.0(25.0)$ & $95.0(15.0)$ & $100.0(15.0)$ & 0.02 & 0.002 & 1.00 \\
\hline EF - median score (interquartile range) & $73.3(38.0)$ & $86.7(20.0)$ & $93.3(20.0)$ & 0.22 & 0.12 & 1.00 \\
\hline $\begin{array}{l}\text { Overall score of MSQv2.1 - median score } \\
\text { (interquartile range) }\end{array}$ & $62.9(27.1)$ & $88.6(18.6)$ & $88.6(18.6)$ & 0.02 & 0.001 & 0.11 \\
\hline
\end{tabular}

${ }^{\dagger}$ Counted more than once; ${ }^{*} P<0.05,{ }^{* *} P<0.01$

EF, emotional function; MSQv2.1, Migraine-Specific Quality of Life Questionnaire version 2.1; NA, not applicable because of a zero value; NRS, numerical rating scale; NSAIDs, nonsteroidal anti-inflammatory drugs; QoL, quality of life; RP, role function - preventive; RR, role function - restrictive

weeks 4 and 8 , the median scores of the RR, RP, and EF domains and the overall score of MSQv2.1 appeared to be stable or just slightly improved, with no significant difference. Clinical testing for sensitivity of the Thai version of MSQv2.1 to change shown in Table 7 demonstrates that improvement in headache severity from moderate to severe to mild correlated significantly with the improvement in the overall QoL score of each of the 2 periods with a correlation coefficient of $0.67-$ 0.77 . Moreover, improvement in headache severity also correlated significantly with the improvement in the RR domain between week 4 and time zero $(r=0.71)$ and between week 8 and time zero $(r=0.71)$ and RP domain between week 4 and time zero $(r=0.77)$ and weeks 8 and $4(r=0.69)$. Change in the proportion of patients taking prophylactic medications between week 8 and time zero correlated significantly with the improvement in the EF domain with a correlation coefficient of 0.66. Median reduction in frequency of attacks 4 weeks and average pain severity score (NRS) between weeks 8 and 4 correlated significantly with the improvement in the EF domain $(r=-0.61)$ and overall QoL score $(r=-0.62)$ (negative correlation was observed between frequency of attacks and EF score and between pain severity score and overall QoL score).

\section{Discussion}

To our knowledge, this is the third reported study for validity and reliability of a non-English version of the MSQv2.1. The first study was conducted in Iran using 106 patients with chronic migraine and episodic migraine to test the validity and reliability of a Persian version of MSQv2.1 in neurology clinics [28]. The investigators reported an internal consistency with Cronbach's $\alpha$ of 0.92 , test-retest reliability with intraclass correlation coefficients of $0.41-0.50$, and convergent validity 
Table 7. Clinical testing for sensitivity of the Thai version of the MSQv2.1 to change of the headache history between 2 periods of 4 weeks

\begin{tabular}{|c|c|c|c|c|}
\hline \multirow[t]{2}{*}{ History of headache } & \multicolumn{4}{|c|}{ Correlation coefficient for median improvement } \\
\hline & $\mathbf{R R}$ & $\mathbf{R P}$ & EF & Overall QoL \\
\hline \multicolumn{5}{|l|}{ Between week 4 and time zero } \\
\hline Median reduction in frequency of attacks 4 weeks before & -0.32 & -0.23 & -0.22 & -0.24 \\
\hline Median reduction in average pain duration per attack, hours & -0.13 & 0.40 & 0.07 & 0.12 \\
\hline Median reduction in average pain severity score (NRS) & 0.24 & 0.16 & -0.33 & 0.16 \\
\hline Improvement in associated symptoms with nausea/vomiting to no nausea/vomiting & -0.12 & -0.18 & 0.31 & -0.09 \\
\hline Improvement in associated symptoms with photophobia/phonophobia to no & 0.32 & 0.09 & 0.27 & 0.29 \\
\hline \multicolumn{5}{|l|}{ photophobia/phonophobia } \\
\hline Change in proportion to take prophylactic medications & 0.24 & -0.21 & 0.25 & -0.21 \\
\hline Improvement in headache severity from moderate to severe to mild & $0.71^{*}$ & $0.77^{* *}$ & 0.40 & $0.77^{* *}$ \\
\hline \multicolumn{5}{|l|}{ Between week 8 and time zero } \\
\hline Median reduction in frequency of attacks 4 weeks before & 0.15 & 0.20 & -0.21 & 0.15 \\
\hline Median reduction in average pain duration per attack, hours & 0.02 & -0.21 & -0.59 & -0.12 \\
\hline Median reduction in average pain severity score (NRS) & 0.34 & 0.17 & 0.01 & 0.28 \\
\hline Improvement in associated symptoms with nausea/vomiting to no nausea/vomiting & 0.33 & 0.13 & -0.16 & 0.25 \\
\hline Improvement in associated symptoms with photophobia/phonophobia to no & 0.00 & 0.24 & -0.24 & 0.06 \\
\hline \multicolumn{5}{|l|}{ photophobia/phonophobia } \\
\hline Change in proportion to take prophylactic medications & -0.45 & -0.26 & $0.66^{*}$ & -0.38 \\
\hline Improvement in headache severity from moderate to severe to mild & $0.71^{*}$ & 0.52 & 0.29 & $0.77^{* *}$ \\
\hline \multicolumn{5}{|l|}{ Between weeks 8 and 4} \\
\hline Median reduction in frequency of attacks 4 weeks before & 0.07 & -0.15 & $-0.61^{*}$ & -0.12 \\
\hline Median reduction in average pain duration per attack, hours & 0.07 & 0.57 & -0.02 & 0.11 \\
\hline Median reduction in average pain severity score (NRS) & -0.39 & -0.50 & -0.35 & $-0.62^{*}$ \\
\hline Improvement in associated symptoms with nausea/vomiting to no nausea/vomiting & 0.15 & 0.15 & 0.03 & 0.22 \\
\hline Improvement in associated symptoms with photophobia/phonophobia to no & 0.20 & -0.05 & 0.40 & 0.30 \\
\hline \multicolumn{5}{|l|}{ photophobia/phonophobia } \\
\hline Change in proportion to take prophylactic medications & -0.50 & -0.30 & 0.20 & -0.40 \\
\hline Improvement in headache severity from moderate to severe to mild & 0.59 & $0.69^{*}$ & 0.26 & $0.67^{*}$ \\
\hline
\end{tabular}

${ }^{*} P<0.05$ and ${ }^{*} P<0.01$

EF, emotional function dimension; MSQv2.1, Migraine-Specific Quality of Life Questionnaire version 2.1; NRS, numerical rating scale; QoL, quality of life; $\mathrm{RP}$, role preventive dimension; $\mathrm{RR}$, role restrictive dimension

to a 36-Item Short Form Survey with a Pearson correlation coefficient of $0.41-0.46$. The second study was conducted in Italy using 182 patients with chronic migraine and a history of medication overuse to test validity and reliability of an Italian version of MSQv2.1 in an inpatient department at a neurology institute [29]. The investigators reported internal consistency with a Cronbach's $\alpha$ of between 0.85 for the EF domain and 0.92 for the RR domain and construct validity to the MIDAS questionnaire with Pearson correlation coefficients with $P<$ 0.001 . The third and present study was conducted in Thailand at a tertiary teaching hospital. The present study tested a Thai version of MSQv2.1 for validity and reliability in addition to evaluating whether it was sensitive to change in clinical practice. Validity, reliability, and sensitivity to change of the Thai version MSQv2.1 were tested in a typical population of migraineurs having female preponderance and a mean age of approximately 40 years with migraine classification of migraine without aura [30-32]. The participants who were recruited were modestly educated $(50 \%-55 \%$ with secondary school education level or less), which might represent the general population in Thailand. The concurrent validity of the Thai version of MSQv2.1 had a significantly negative correlation with almost all the migraine characteristics except associated symptoms of migraine, which was not correlated with any of the dimension scores or overall QoL score of the Thai version of MSQv2.1. A reason for the lack of correlation between these associated symptoms and the Thai of version MSQv2.1 was that the proportion of patients who had no associated symptoms accounted for $50 \%$ of the participants. During evaluations of 2-week intervals, test-retest reliability of the history of headache was nearly the same for frequency of attacks, average pain duration and pain severity, associated symptoms, and headache severity on each occasion. The test-retest reliability of the Thai version of MSQv2.1 using 
Spearman's correlation coefficient was strong $(0.60-0.79)$ among almost all the items with the exception of items 2 and 14 that were rated moderate but acceptable (correlation 0.40 0.59) [33]. The strong correlation of the test-retest reliability might be the inclusion criteria for the test. This study included people who thought that their QoL was the same as they had in the previous 2 weeks. However, the test-retest reliability was used to measure variability of respondents. If participants had a different QoL from the previous 2 weeks, it might not measure the variability between the respondents but measure the condition changed. By contrast, if the study included respondents who thought that they had the same QoL in the 2 periods, less variability would be detected. The internal consistency of the questionnaire was excellent according to Cronbach's $\alpha$ value of $\geq 0.9$ [34]. However, this excellent internal consistency might be an overestimation because of the small sample size. Considering the test for sensitivity of the Thai version of MSQv2.1 to change, the history of headache, especially the frequency of attacks, average pain duration per attack, pain severity score (NRS), headache severity, associated symptoms of nausea or vomiting, photophobia or phonophobia, and taking prophylactic medications, improved or increased over the time of follow-up with some reaching significant differences but others not. The median scores of the RR, RP, and EF dimensions and the overall QoL score improved over time with some reaching significant difference but others not. These differences that did not reach significance might be the result of a sample size insufficient to detect a true difference. Those differences that did reach a significance would represent a true difference. An improved history of headache and QoL would be the effect of a prophylactic medication that could reduce frequency of headache and pain severity leading to reduced headache severity and might cause headache to respond to acute pain medications [35] with an effect starting at the first 4-week period and then increasing in the following 2-3 months [36]. Data in this study to support the headache response to the acute pain medication were that no patient took migraine-specific medications in week 8 and the proportion of those who reported effectiveness of the acute pain medication were higher starting at week $4(82 \%)$. The sensitivity of the Thai version of MSQv2.1 to change is given in Table 7. Reduction in frequency of attacks and pain severity score (NRS) and improvement in headache severity correlated significantly with an improvement in each dimension and overall QoL score of the Thai version of MSQv2.1. Some improvement, but not correlated with the QoL, may be a result of inadequate sample size. Interestingly, a change in taking prophylactic medication improved the EF domain that lowered frequency of attacks and might have made patients feel confident with the efficacy of the prophylactic medications and less worried about occurrence of future headache.
This study was limited by its small sample size for testing of internal consistency and sensitivity to change, lack of correction of $P$-values for multiple analyses, and analysis of test-retest reliability for those who thought their QoL was the same, which might reduce variability to less than it should be.

\section{Conclusion}

The Thai version of MSQv2.1 had concurrent validity, acceptable internal consistency, moderate-to-strong test-retest reliability, and strong correlation between improvement in headache severity and overall QoL score. The Thai version of MSQv2.1 would be helpful to measure clinical outcomes in clinical practice. A future study using a larger sample size and longer follow-up is required to fully test internal consistency and sensitivity to change.

Author contributions. TA, PV, JN, and KP contributed substantially to the conception and design, acquisition, analysis, and interpretation of data. All authors were involved in drafting the manuscript and critically revising it. All authors approved the final version submitted for publication and take responsibility for the statements made in the manuscript.

Acknowledgments. We thank the participants and Christopher Bell, USP Health Outcomes, GSK, for permitting to translate the English version of MSQv2.1 into Thai version and to use the Thai translation in a study to evaluate the Thai version of MSQv2.1.

Conflict of interest statement. All authors have completed and submitted the ICMJE Uniform Disclosure Form for Potential Conflicts of Interest. None of the authors disclose any conflict of interest.

\section{References}

[1] Headache Classification Committee of the International Headache Society (IHS). The International classification of headache disorders, 3rd edition (beta version). Cephalalgia. 2013; 33:629-808.

[2] Dahlöf CG. Measuring disability and quality of life in migraine. Drugs Today (Barc). 2003; 39 Suppl D:17-23.

[3] Freitag FG. The cycle of migraine: patients' quality of life during and between migraine attacks. Clin Ther. 2007; 29:939-49.

[4] Clouse JC, Osterhaus JT. Healthcare resource use and costs associated with migraine in a managed healthcare setting. Ann Pharmacother. 1994; 28:659-64.

[5] Cavallini A, Micieli G, Bussone G, Rossi F, Nappi G. Headache and quality of life. Headache. 1995; 35:29-35. 
[6] Wagner TH, Patrick DL, Galer BS, Berzon RA. A new instrument to assess the long-term quality of life effects from migraine: development and psychometric testing of the MSQOL. Headache. 1996; 36:484-92.

[7] Jhingran P, Osterhaus JT, Miller DW, Lee JT, Kirchdoerfer L. Development and validation of the Migraine-Specific Quality of Life Questionnaire. Headache. 1998; 38:295-302.

[8] Kosinski M, Bayliss MS, Bjorner JB, Ware JE Jr, Garber WH, Batenhorst A, et al. A six-item short-form survey for measuring headache impact: the HIT-6. Qual Life Res. 2003; 12:963-74.

[9] Manhalter N, Bozsik G, Palásti A, Csépány E, Ertsey C. The validation of a new comprehensive headache-specific quality of life questionnaire. Cephalalgia. 2012; 32:668-82.

[10] Langeveld JH, Koot HM, Passchier J. Headache intensity and quality of life in adolescents. How are changes in headache intensity in adolescents related to changes in experienced quality of life? Headache. 1997; 37:37-42.

[11] Hartmaier SL, Santanello NC, Epstein RS, Silberstein SD. Development of a brief 24-hour Migraine-Specific Quality of Life Questionnaire. Headache. 1995; 35:320-9.

[12] Jhingran P, Davis SM, LaVange LM, Miller DW, Helms RW. MSQ: migraine-specific quality-of-life questionnaire. Further investigation of the factor structure. Pharmacoeconomics. 1998; 13:707-17.

[13] Martin BC, Pathak DS, Sharfman MI, Adelman JU, Taylor F, Kwong WJ, et al. Validity and reliability of the Migraine-Specific Quality of Life Questionnaire (MSQ Version 2.1). Headache. 2000; 40:204-15.

[14] Pathak D, Martin B, Kwong J, Batenhorst A. Evaluation of the Migraine-Specific Quality of Life Questionnaire (MSQ version 2.0) using confirmatory factor analysis. Qual Life Res. 1998; 7:647.

[15] Bagley CL, Rendas-Baum R, Maglinte GA, Yang M, Varon SF, Lee J, et al. Validating Migraine-Specific Quality of Life Questionnaire v2.1 in episodic and chronic migraine. Headache. 2012; 52:409-21.

[16] Cole JC, Lin P, Rupnow MF. Validation of the Migraine-Specific Quality of Life Questionnaire version 2.1 (MSQ v. 2.1) for patients undergoing prophylactic migraine treatment. Qual Life Res. 2007; 16:1231-7.

[17] Dodick DW, Silberstein S, Saper J, Freitag FG, Cady RK, Rapoport AM, et al. The impact of topiramate on health-related quality of life indicators in chronic migraine. Headache. 2007; 47:1398-408

[18] Silberstein SD, Lipton R, Dodick D, Freitag F, Mathew N, Brandes $\mathrm{J}$, et al. Topiramate treatment of chronic migraine: a randomized, placebo-controlled trial of quality of life and other efficacy measures. Headache. 2009; 49:1153-62.

[19] Yang M, Rendas-Baum R, Varon SF, Kosinski M. Validation of the Headache Impact Test (HIT- $6^{\text {mD }}$ ) across episodic and chronic migraine. Cephalalgia. 2011; 31:357-67.

[20] Rendas-Baum R, Yang M, Varon SF, Bloudek LM, DeGryse RE, Kosinski M. Validation of the Headache Impact Test (HIT-6) in patients with chronic migraine. Health Qual Life Outcomes. 2014; 12:117.
[21] Diener HC, Bussone G, Van Oene JC, Lahaye M, Schwalen S, Goadsby PJ, et al. Topiramate reduces headache days in chronic migraine: a randomized, double-blind, placebo-controlled study. Cephalalgia. 2007; 27:814-23.

[22] Cady R, Schreiber C. Botulinum toxin type A as migraine preventive treatment in patients previously failing oral prophylactic treatment due to compliance issues. Headache. 2008; 48:900-13.

[23] De Diego EV, Lanteri-Minet M. Recognition and management of migraine in primary care: influence of functional impact measured by the headache impact test (HIT). Cephalalgia. 2005; 25:184-90.

[24] Nachit-Ouinekh F, Dartigues JF, Henry P, Becg JP, Chastan G, Lemaire N, et al. Use of the headache impact test (HIT-6) in general practice: relationship with quality of life and severity. Eur J Neurol. 2005; 12:189-93.

[25] Classification and diagnostic criteria for headache disorders, cranial neuralgias and facial pain. Headache classification committee of the International Headache Society. Cephalalgia. 1988; 8 Suppl 7:1-96.

[26] Headache Classification Subcommittee of the International Headache Society. The international classification of headache disorders, second edition. Cephalalgia. 2004; 24 Suppl 1:1-160.

[27] Siegel S, Castellan NJ Jr, editors. Nonparametric statistics for the behavioural sciences. 2nd ed. Boston MA: McGraw Hill; 1988, p. 235-44.

[28] Zandifar A, Masjedi SS, Haghdoost F, Asgari F, Manouchehri $\mathrm{N}$, Banihashemi M, et al. The psychometric properties of the Persian Migraine-Specific Quality Of Life Questionnaire version 2.1 in episodic and chronic migraines. Sci World J. 2013; 2013:950245.

[29] Raggi A, Giovannetti AM, Schiavolin S, Leonardi M, Bussone G, Grazzi L, et al. Validating the Migraine-Specific Quality of Life Questionnaire v2.1 (MSQ) in Italian inpatients with chronic migraine with a history of medication overuse. Qual Life Res. 2014; 23:1273-7.

[30] Smitherman TA, Burch R, Sheikh H, Loder E. The prevalence, impact, and treatment of migraine and severe headaches in the United States: a review of statistics from national surveillance studies. Headache. 2013; 53:427-36.

[31] Kim BK, Chung YK, Kim JM, Lee KS, Chu MK. Prevalence, clinical characteristics and disability of migraine and probable migraine: a nationwide population-based survey in Korea. Cephalalgia. 2013; 33:1106-16

[32] Phanthumchinda K, Sithi-Amorn C. Prevalence and clinical features of migraine: a community survey in Bangkok, Thailand. Headache. 1989; 29:594-7.

[33] Spearman's correlation Page. [online]. 2016 [cited 2016 Jun 22]. Available from: http://www.statstutor.ac.uk/resources/uploaded/ spearmans.pdf

[34] George D, Mallery P, editors. SPSS for Windows step by step: a simple guide and reference (11.0 update). 4th ed. Boston: Allyn and Bacon; 2003

[35] Silberstein SD. Preventive migraine treatment. Continuum (Minneap Minn). 2015; 21:973-89.

[36] Silberstein SD. Topiramate in migraine prevention: a 2016 perspective. Headache. 2017; 57:165-78. 